\title{
Making Decisions with Reactive Multi-agent Systems: A Possible Alternative to Regular Decision Processes for Platoon Control Issue
}

\author{
Baudouin Dafflon and Franck Gechter \\ IRTES-SET, UTBM, Belfort, \\ France \\ franck.gechter@utbm.fr, baudouin.dafflon@utbm.fr
}

\begin{abstract}
Decision processing is a key element in computer science, in automatic control and in robotics. The literature presents a lot of various approaches for decision processing. These approaches generally depend on both the adopted conceptual point of view and of the application field. Some of the classical methods suffers from several problems such as a limited adaptivity, or a high computational cost. In this context, reactive multi-agent systems can be good candidates to overcome some of these drawbacks. The goal of this paper is to present how to use reactive multi-agent systems to make decision, what are their advantages and their drawbacks as compared to classical methods. It also illustrates the proposal with an example of application dealing with the platoon control issue.
\end{abstract}

Keywords: Reactive multi-agent systems, platoon control, vehicle control

\section{Introduction}

Decision processing is a key element in computer science, in automatic control and in robotics. It can be defined as the process of choosing one action or a sequence of actions aimed at reaching one goal while respecting constraints (energy consumption, kinematic and dynamical limits, etc.). Thus, a good decision process must take into account both the problem constraints and the best way to reach system's goal. In some cases the decision process can be composed of several steps [21]: (1) Definition of the problem; (2) Definition of the goal(s); (3) Problem solving process; (4) Evaluation of the obtained solutions; (5) Selection of one solution and application of the related actions. In terms of mobile robotics the goal can have different definitions depending on the level of abstraction that is used. One can find high level definitions such as gathering specific information, collecting a specified amount of stones in mars rover mission or low level definitions such as reaching one position, performing one specific action, etc. 
In literature, one can find a lot of various approaches for decision process. Among the most widespread, one can cite artificial neural networks [16] the fuzzy logic [19], or the stochastic approaches such as Markov decision processes [26] or those based on Bayesian networks. Of course, the literature contains also multi-agent based problem solving methods and decision processes such as [25].

The classical methods suffers from several problems which, even if they are acceptable in most cases, can be dissuasive for some applications. For instance, neural networks have limited adaptation skills due to the way the neural weights are computed/learned, Markov decision processes require a large amount of knowledge to be usable (transition function, set of states, set of actions, reward function, etc.) and suffers from a high computational cost (generally in $\left.o\left(n b r_{\text {states }}^{2}\right)\right)$ making them hard to use for multi-robot applications in dynamical environment, etc.

Since a couple of years multi-agent systems, have became more and more wide-spread for a wide range of applications (simulation, problem solving, complex system modelling, etc.) in computer science and especially in artificial intelligence. Their properties (adaptability, robustness, etc.) can make them good candidates for developing new kinds of decision processes.

A multi-agent system can be defined as a set of entities, named agents, in interaction with each-other and with their evolution area, generally named environment, and aimed at producing collectively a spatio-temporal organization. The main difficulty while developing such systems is prediction and the verification of the organizational global properties while having knowledge on only local agents behaviours and interaction mechanisms. In literature, two principal trends can be encountered: the cognitive and the reactive approaches. The cognitive trend relies on the interactions between entities with high level reasoning, behavioural and/or perceptual abilities that can be qualified to be intelligent. By contrast, the reactive approach focuses on agents interactions instead of their intrinsic capacities. The main interest of this kind of approaches is this ability to adapt to a wide range of domain while keeping a conceptual and functional simplicity of the programmed entities. These models are well adapted to simulation/modelling of complex systems and to dynamic problem solving methods. They are used in various application fields such as artificial life simulation [12], multi-vehicle navigation [15], etc. As for the interaction models, several inspiration sources can be found in literature. Among the most widespread, one can cite the biological inspired approaches and the models based on Physics. The first ones can be based on the use of artificial pheromons or on the adaptation of biological behaviours on specific problems [3]. The Physics inspired approaches are based whether on regular forces [6] or force fields [2].

Using a reactive multi-agent system for building a decision process is, in some cases, natural especially when the initial problem to solve is already distributed on the functional or topological points of view or when some elementary coupling between problem entities are already known. Moreover, literature contains also several conception methodologies/principles among which one can cite [4], [22], etc. Even if some of them are hard to be adapted to a wide range of problems, 
it is possible to extract from them guidelines for conceiving such systems. As for the reactive systems, the task is hard to tackle with due to their complexity (linked to the huge number of entities in interaction) and to the emergent and non-predictible aspect of the global properties. Thus, conceiving a reactive multiagent system for decision purpose implies to focus on interactions and on the way they are put into practice (directly or indirectly through environment) instead on focusing on agent themselves. More over, one must also define how the link between the problem and the agency is made and how to convert emergent properties evaluation into a suitable set of actions. The general structure of a reactive multi-agent decision process can be represented by Figure 1.

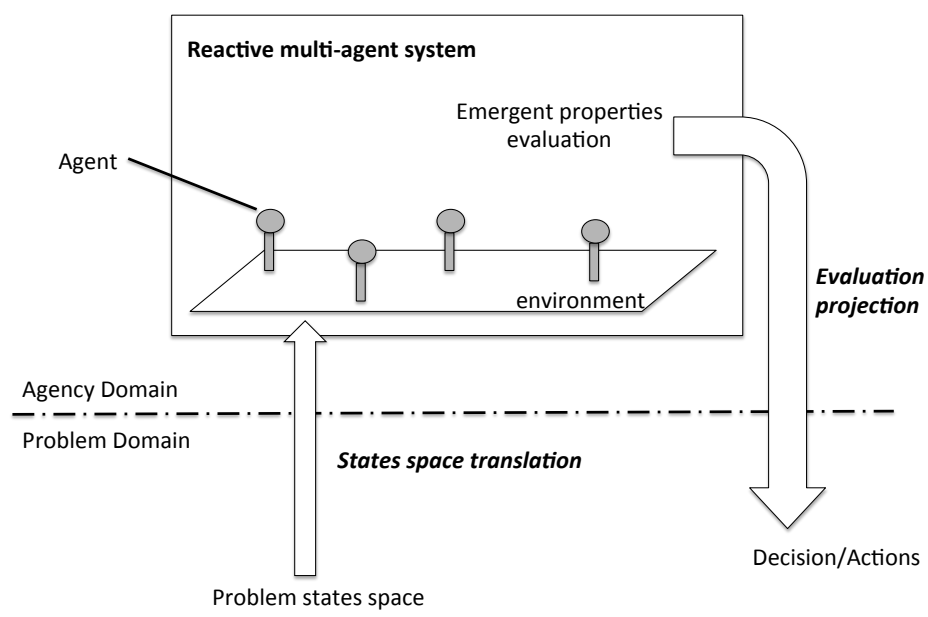

Fig. 1. General description of a reactive multi-agent decision process.

The goal of this paper is to explain how reactive multi-agent systems can be used to make decision and what kind of advantages (and drawbacks) they can provide as compared to regular methods. To that way, we will give a description of the key elements (problem interpretation, problem solving and action(s) selection) that have to be taken into consideration to tackle this kind of issue. These descriptions will be illustrated by an example dealing with the platoon control issue.

The paper is structured as follows: Section 2 gives a global presentation of the key elements required for reactive multi-agent system decision processes. This section details, in particular, the role of the environment (Section 2.1) and how the problem and its characteristics/constraints can be translated into this structure common to all agents, the role of the interactions and how they can 
be chosen (Section 2.2) and how to convert the agency emergent properties into suitable actions for the system (Section 2.3). This section is concluded by a presentation of the most widespread application fields and of the limitations of the reactive approach. Section 3 shows an instanciation of the approach to the platoon control issue. Finally, Section 4 concludes this paper giving the main limitations encountered with these new kind of approaches and giving some clues for further works.

\section{Key Elements for Reactive Multi-Agent Systems for Decision Process}

\subsection{Agent Environment: An Active Element of the Decision Process}

The role of the environment is particularly important in multi-agent systems designed for decision processing. Generally, this one is built so as to "translate" the problem into the agents world. This translation relies on a projection of space state of the problem into a space state which can be travelled by the agents.

Since several years, works on multi-agent systems give a more and more active role to the environment especially when agents are situated [23]. In such systems, the environment is an active entity with its own behaviours. Thus, it can handle various role such as shared memory, interaction catalyst or coordination entity as in pheromon based approach or stigmergic approaches.

Several models for environment can be used. [20] proposes an environment which defines perception/action relations with agents. Environments can be whether centralized or decentralized. Finally, in some cases, the environment can be defined as a standalone multi-agent system [12].

In the decision making context, the environment has got the central role. Since the agents behaviours are as simple as possible, the system can compute only thanks and in the environment. As compared to classical decision making approaches, the main interest of this active environment is to be able to link together the problem world and the solving process world. Besides, since the environment has got its own behaviours, it can adapt, while maintaining a temporal coherence of the information on the problem, to the dynamical evolution of it. This dynamical adaptation cannot be made in such a wide extend with Markov decision processes or in artificial neural networks.

\subsection{Agents Interactions: A Conceptual Choice}

An interaction is defined as a dynamical relation between agents through a set of mutual actions. These actions can have an influence on other agents perception and then on their further actions. The main difference with classical systems is the importance of this interaction that prevails on the definition of the agents themselves. These inter-agent links can be direct or indirect using the environment as in pheromon based approaches. Two main inspiration sources are generally used for defining interactions: Biology or Physics. 
Biological approaches relies on the study of the behavioural mechanisms of social insects. Among the most widespread, one can cite ant based algorithms which relies on artificial pheromons dropped into environment and aimed at sharing information at global level [11]. Other models, inspired by wolves, fireflies or social spiders have been also be used in several context. The main interest of bio-inspired approaches is the fact that the global emergent organisation is known in advance. Thus, the engineering strategy relies on an adaptation of existing and already observed behaviours to a specific decision process. The decision is obtained when an equilibrium between an amplification mechanism and a regulation mechanism is reached. The decision relies then on the interpretation of the information persistence into environment such as the geometrical repartition of agents.

Physics inspired approaches are also widespread. As opposed to biological inspiration, the engineering methodology is not based on the global expected result but on the definition of the behaviour one want to provide to agents. These methods generally relies on attraction/repulsion mechanisms. As in biological paradigm, the goal is to obtain a stable or meta-stable state of equilibrium which can take the form of a specific spatial organization of agents.

To summarize, the goal of the interaction mechanisms, whether they are Bio or Physics inspired, is to obtain a spatio-temporal organization that corresponds to the result of the problem space state exploration by the agents. When a perturbation occurs, the system evolves into another organization which corresponds to the new available data on the problem.

\subsection{Evaluating Agency Properties: the Key for a Good Decision}

Agents, Complex Systems, Emergence and Self-Organization The evaluation of the agency properties is the most difficult part to handle due the the complex character of reactive multi-agent systems. A complex system is considered as a set of components in interaction, the global behaviour of which, and its evolution, can neither be computed nor predicted by an external observer. Thus a system is said to be complex if the global final result can be predicted only by experiments or simulations even if all components and internal relations are perfectly known. Reactive multi-agent systems can be considered in some ways as complex systems. Even if the internal local mechanisms are perfectly known, the application of them by each agent, depending on their perception and behaviours, lead to a dynamic un-predictible variation of the agency properties. These considerations allow to differentiate standard particle systems from particle argent systems (i.e. systems in which agents acts as particles, the behaviours of which change in run-time relatively to their perception).

The problem of the global evaluation has to be put in parallel with emergence and self-organizing notions that lead to numerous publications on both practical and theoretical levels. Self-organization is generally linked to an increase in the order of the system without any possible external control of the process that lead to this result. In [9], self-organizing property is defined as a "dynamical and adaptive process allowing the system to acquire or maintain a structure without 
any external control". If the self-organization ability of one system is defined with a consensus in the scientific community, the definition of emergence is far from being as clear. The common sense is linked to the presence of an external observer able to detect, measure and analyse global phenomena produced by a process. Emergence leads to many works in computer science [10], complex systems studies [17], in sociology, etc. As the study of the complex system cannot be performed in a reductionist frame, the emergence leads to the same kinds of issues. Thus, it can not be considered as a system property but as a property of the point of view one can have on the system. In [9], a system is defined to have emergent properties when phenomena appear dynamically at a macroscopic level thanks to the microscopic actions that link the system components together.

Due to the complexity of such systems and to the difficulties encountered to define the emergence, this part is sometimes hard to tackle. The evaluation of the emergent properties is generally split into two steps: (1) Developing a pertinent measure of the agency properties; (2) Projecting this measure into the possible actions space.

Evaluating Emergent Properties The evaluation of emergent properties allows to have a pertinent analysis of the solution given by the multi-agent system. In many examples, this relies on a simple observation of the global result. In this case, the engineering process is similar to a reinforcement learning such as the one that can be found in artificial neural networks. Once the system is built, a tuning phase of the local parameters, generally made empirically taking into account system goal, has to be done. Some systems are also based on more precise evaluation functions that are not necessary tied directly to the problem domain. These functions take into account both local and global properties. The literature proposes solutions inspired by Biology (fitness function), Sociology (utility function, satisfaction, altruism, etc.) or Physics (entropy, energy, etc.)

Once the evaluation is made, the projection function can be defined relying on mathematical considerations and problem knowledge.

\subsection{Application Fields, Properties and Difficulties}

The global structure and the different steps of a multi-agent decision process have been sketched into the previous sections. Until now, multi-agent decision processes applied to robotics can be found scarcely in literature. They have been used for multi-configuration platoon control [15], [18], obstacle avoidance [24], driving assistance [13] or automatic parking device [7].

The properties are mostly tied to the multi-agent aspect of these systems such as adaptability or robustness. For instance, multi-agent based obstacle avoidance algorithms allow to deal with one fixed obstacle but also with several moving obstacles without changing any elements of the system. By the same, platoon control algorithms are able to adapt the platoon structure according to vehicles/robots perceptions. Moreover, the number of agents can be adapted to the available computational resources providing a decision process with a controllable complexity independently to the dynamical variations of the problem. 
If we assume that the local interactions are aimed at maintaining an equilibrium between problem variations (translated into agents environment) on one side and the goal of the system on the other, the two main difficulties are in the translation of the problem space state into a suitable space environment for agents and the interpretation of the emergent properties/structure as a decision. The first difficulty is mainly linked to the dimension of the problem states space and the desired dimension for the multi-agent system environment. The second problem is more delicate since the projection of the agency properties relies mainly on a definition of a "good" estimation function. This function has to takes into account agents interactions, emerging structure, problem domain, etc. Unfortunately, there is no method to design such a function for the moment.

\section{Application to Platoon Control}

The goal of this section is to present how to instantiate the preceding proposals within a concrete example. After a state of the art of the platoon control issue, we will explain how to apply the previous statements to a platoon of vehicles.

\subsection{State of the Art}

A platoon is generally considered as a set vehicles moving synchronously following a predefined geometrical configuration (column, line, echelon, etc.) without any material coupling. Platoon control methods are generally divided into two categories: global and local. In global approaches, vehicles are controlled by a system which relies on information shared by vehicles on the convoy level. Global approaches mostly requires a vehicle to vehicle (V2V) and/or a vehicle to infrastructure (V2I) communication system. For instance, [1] proposes a system where vehicles are represented by a bicycle cinematic model. Lateral and longitudinal control are separated. Lateral control is made using a shared reference trajectory. Longitudinal control is made using the curvilinear abscissa of the leader vehicle over the reference trajectory. In [14] an algebraic graph theory is used. The vehicles exchange information according to a pre-specifies communication graph. The feedback control is based only on relative information about vehicle states shared via the communication links. By contrast, local approaches are based on reactive capability of vehicles. Vehicle actions are based on its own perceptions. In [8], for instance, an automatic control mechanism based on the PID controller has been proposed. Longitudinal control consists in maintaining a time $T=\frac{D}{V}$ constant between two vehicles, where $D$ is the distance between two vehicles and $v$ is the vehicle speed. By contrast, lateral control consists in maintaining the direction angle between the vehicle and its predecessor close to zero. [5] presents local approaches based on a virtual mechanical link inspired by physics. Each vehicle is considered to be able to perceive and reacts while maintaining a constant distance with its predecessor using an impedance control model composed of springs and dampers. 
In this paper, a local approach for platoon system is presented using the reactive multi-agent system decision process exposed in the previous section. In this example, each vehicle has got its own multi-agent process for decision making.

\subsection{Applying Reactive Agent Decision Process to Platoon Control}

Platoon system could be considered as a trajectory following problem. By contrast to classical methods, the trajectory to follow is defined by the leader vehicle instead of being known in advance.

Building the Environment The agents' environment is built using data from the problem. In the platoon control context, the data is composed of the position of the leader, relatively to the considered follower vehicle, and its path. The agents' environment is then considered as a 2D space where the leader trajectory is translated into an attractive area and where the other region of the vehicle space are set to be repulsive (see Figure 2).

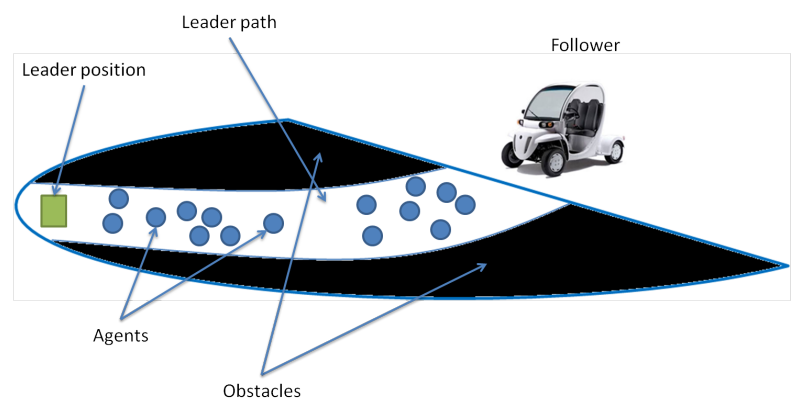

Fig. 2. Translation of the problem data into the agents' environment.

Interaction model Interactions in agents' environment can be summarized as shown in Figure 3.

- Agent-Leader representative interaction (Attraction) : The attraction force generated by the leader representative is computed as a linear force defined by:

$$
\boldsymbol{F}=\beta_{g} m \boldsymbol{A}_{\boldsymbol{i}} \boldsymbol{T}
$$

- Agent-Obstacles interaction (Repulsion) : The repulsion is made by classical newtonian force in $\frac{1}{r^{2}}$.

$$
\left\{\begin{array}{l}
F o_{i}^{X}=\sum_{o}\left(\Delta_{o} \cdot m \cdot m_{o} \frac{\left(x_{i}-x_{o}\right)}{\left(\left(y_{i}-y_{o}\right)^{2}+\left(x_{i}-x_{o}\right)^{2}\right)^{\frac{3}{2}}}\right) \\
F o_{i}^{Y}=\sum_{o}\left(\Delta_{o} \cdot m \cdot m_{o} \frac{\left(y_{i}-y_{o}\right)}{\left(\left(y_{i}-y_{o}\right)^{2}+\left(x_{i}-x_{o}\right)^{2}\right)^{\frac{3}{2}}}\right)
\end{array}\right.
$$


- Agent-Agent interaction (Repulsion) : This interaction shares the same formulation as agent-obstacle repulsion. The repulsion between agents is generally introduced to ensure a homogeneous exploration of the environment avoiding false agents grouping and local minima locking. This repulsion If $A_{i}$ and $A_{j}$ are two agents located in $P_{i}$ and $P_{j}$, the repulsion force is given by:

$$
\boldsymbol{F} \boldsymbol{r}_{\boldsymbol{i j}}=\alpha m_{i} m_{j} \frac{\boldsymbol{P}_{\boldsymbol{i}} \cdot \boldsymbol{P}_{\boldsymbol{j}}}{\left\|\boldsymbol{P}_{\boldsymbol{i}} \cdot \boldsymbol{P}_{\boldsymbol{j}}\right\|^{3}}
$$

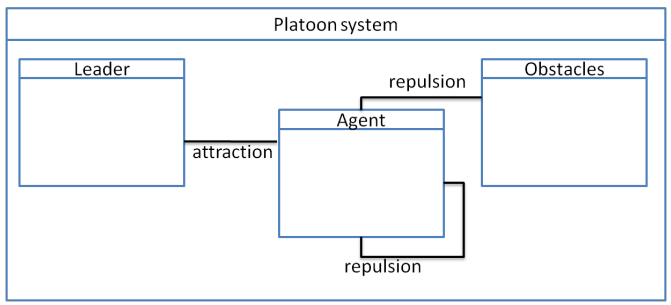

Fig. 3. Interaction diagram.

Making the Decision As previously explained, the decision making process is based on agent's population observation. So as to select the tuple (steering angle, speed) which correspond to a valid order for the vehicle low level controller, one makes an observation of the agency based on topological indicators. Two vectors are computed. The first, called $P_{\text {dir }}$ is the vector which starts from the representative of the follower vehicle (generally taken as the origin of the agents' environment) and finishes to the mean position of the agents. The second denoted $P_{\text {speed }}$ is composed of the mean speed of all agents. A combination of these two vectors leads to a set point to sent to the vehicle and denoted $P_{\text {order }}$ (see Figure 4).

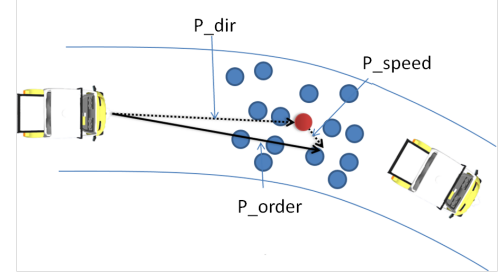

Fig. 4. Decision making. 


\subsection{Results}

So as to test the approach, simulations have been performed considering lateral and longitudinal distances.

Lateral Distance Lateral distance measures the distance between leader and follower trajectories. This distance should always be nearest as possible the setpoint. Figure 5 (top) shows the distance error. Taking account time stabilization, the maximal error is around 0.15 meter.

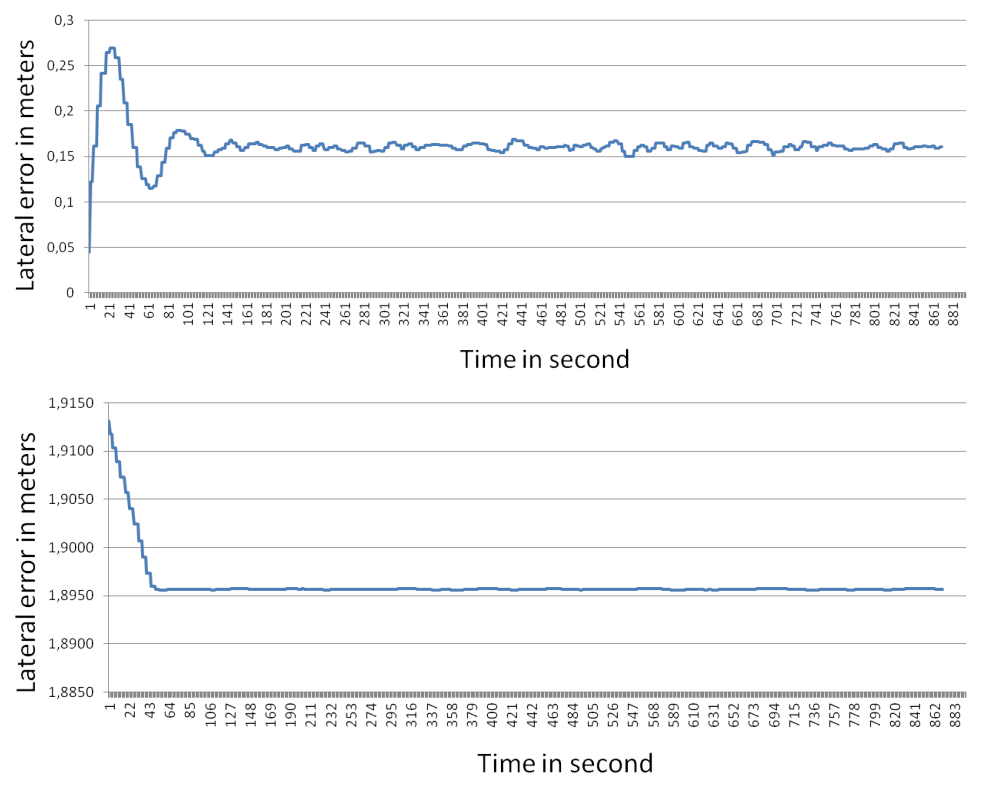

Fig. 5. Lateral distance error (top) and longitudinal distance (bottom).

Longitudinal Distance Longitudinal distance consists of mesuring distance between tow vehicles. This distance should always be above a security distance and nearest as possible the setpoint. Security distance is set to 1 meter. Figure 5 (bottom) shows this distance during simulation. Longitudinal distance is always over security distance and oscillations are mastered.

\section{Conclusion and Perspectives}

Using multi-agent system as a decision process is an approach which can be considered as a good alternative to classical decision process methods. Indeed, it 
is able to provide interesting properties such as adaptability and robustness and can deal by nature with dynamical problems. By contrast, due to its novelty, this approach is harder to use. The principal difficulties in conception are the translation of the system states space into a suitable environment that can be travelled by agents and the projection function which evaluates agency emergent properties and translates them into decisions.

Its application to the platoon issue contrasts with the classical agent methods which consider each vehicle as an agent. In this example, each vehicle uses a system of agents which allows to make a suitable decision taking into account problem constraints into an abstract agent's environment and chossing one action using agency properties evaluation. The other first examples found in literature give also some good results. For the moment, they are limited to situated problems where problem dimension fit to the dimension of the decision space. It seems now interesting to perform further research in this field so as to develop rigorous conception methodologies and to test this new kind of approaches to other application fields.

\section{References}

1. Avanzini, P., Thuilot, B., Martinet, P.: A control strategy taking advantage of inter-vehicle communication for platooning navigation in urban environment. In: Workshop "Perception and Navigation for Autonomous Vehicles in Human Environment" at IEEE/RSJ International Conference on Intelligent Robots and Systems (IROS). San Francisco (USA) (2011)

2. Bautin, A., Simonin, O., Charpillet, F.: Stratégie d'exploration multirobot fondée sur les champs de potentiels artificiels. Revue d'Intelligence Artificielle 26(5), 523$542(2012)$

3. Bourjot, C., Chevrier, V., Thomas, V.: How social spiders inspired an approach to region detection. AAMAS pp. 426-433 (2002)

4. Burrafato, P., Cossentino, M.: Designing a multi-agent solution for a bookstore with the passi methodology. In: AOIS@CAiSE (2002)

5. Contet, J.M., Gechter, F., Gruer, P., Koukam, A.: Reactive multi-agent approach to local platoon control: stability analysis and experimentations. International Journal of Intelligent Systems Technologies And Application. (2011)

6. Contet, J.m., Gechter, F., Lefoulon, L.: Autonomous emergent painting triggered by music and motion. International Journal of Arts and Technology (2013)

7. Dafflon, B., Contet, J.m., Gechter, F., Gruer, P.: Toward a reactive agent based parking assistance system. In: International Conference on Tools with Artificial Intelligence (2012)

8. Daviet, P., Parent, M.: Longitudinal and lateral servoing of vehicles in a platoon. IEEE Intelligent Vehicles Symposium, Proceedings pp. 41 - 46 (1996)

9. De Wolf, T., Holvoet, T.: Emergence versus self-organisation: different concepts but promising when combined. Engineering Self Organising Systems: Methodologies and Applications, Lecture Notes in Computer Science 3464, 1-15 (2005)

10. Dessalles, J.L., Ferber, J., Phan, D.: Emergence in Agent based Computational Social Science: Conceptual, Formal and Diagrammatic Analysis. In: Intelligent Complex Adaptative Systems, p. 24 (2008) 
11. DiMarzo-Serugendo, G., Karageorgos, A., Rana, O., Zambonelli, F.: Engineering Self-Organising Systems: Nature-Inspired Approaches to Software Engineering. Lecture notes in Atificial intelligence, number 2977 ISBN-3540212019 (2004)

12. Galland, S., Gaud, N., Demange, J., Koukam, A.: Environment model for multiagent-based simulation of 3D urban systems. In: the 7th European Workshop on Multiagent Systems (EUMAS09). Ayia Napa, Cyprus (2009)

13. Gechter, F., Contet, J.m., Gruer, P., Koukam, A.: Car-driving assistance using organization measurement of reactive multi-agent system. Procedia Computer Science CS pp. 317-325 (2010)

14. Lafferriere, G., Caughman, J., Williams, A.: Graph theoretic methods in the stability of vehicle formations. In: American Control Conference. vol. 4, pp. 3729-3734 (2004)

15. Lozenguez, G., Adouane, L., Beynier, A., Mouaddib, A.I., Martinet, P.: Calcul distribué de politiques d'exploration pour une flotte de robots mobiles. In: JFSMA. pp. $117-126(2011)$

16. Matsuda, S.: A neural network model for the decision-making process based on anp. In: Neural Networks, 2006. IJCNN '06. International Joint Conference on. pp. 1421-1426 (2006)

17. Moncion, T., Amar, P., Hutzler, G.: Automatic characterization of emergent phenomena in complex systems. Journal of Biological Physics and Chemistry 10, 16-23 (2010)

18. Mouad, M., Adouane, L., Schmitt, P., Khadraoui, D., Martinet, P.: Mas2car architecture - multi-agent system to control and coordinate teamworking robots. In: ICINCO (2). pp. 451-456 (2011)

19. Naim, S., Hagras, H.: A hybrid approach for multi-criteria group decision making based on interval type-2 fuzzy logic and intuitionistic fuzzy evaluation. In: Fuzzy Systems (FUZZ-IEEE), 2012 IEEE International Conference on. pp. 1-8 (2012)

20. Russell, S.J., Norvig, P.: Artificial Intelligence - A Modern Approach (3. internat. ed.). Pearson Education (2010)

21. Schneider, D.K.: Modlisation de la dmarche du dcideur politique dans la perspective de l'intelligence artificielle. Thse de l'Unit Technologie de Formation et Apprentissage - Facult de Psychologie et des Sciences de l'Education - Universit de Genve (1994)

22. Simonin, O., Gechter, F.: An environment-based methodology to design reactive multi-agent systems for problem solving. In: (Lecture Notes in Artificial Intelligence) LNAI Vol. 3830 : Environment for Multiagent Systems II (revised and selected papers of Environment for Multi Agent Systems (E4MAS) 2005). pp. 32-49. Springer-Verlag Berlin Heidelberg (2006)

23. Weyns, D., Parunak, H.V.D., Michel, F., Holvoet, T., Ferber, J.: Environments for multiagent systems state-of-the-art and research challenges. In: E4MAS. pp. 1-47 (2004)

24. Yang, S., Gechter, F., Koukam, A.: Application of reactive multi-agent system to vehicle collision avoidance. In: The 20th IEEE International Conference on Tools with Artificial Intelligence ICTAI, Dayton, Ohio, USA. pp. 197-204. IEEE Computer Society (2008)

25. Zhao, P., Suryanarayanan, S., Simoes, M.: An energy management system for building structures using a multi-agent decision-making control methodology. In: Industry Applications Society Annual Meeting (IAS), 2010 IEEE. pp. 1-8 (2010)

26. Zhou, Y., Ma, L., Mathew, J., Sun, Y., Wolff, R.: Maintenance decision-making using a continuous-state partially observable semi-markov decision process. In: Prognostics and Health Management Conference, 2010. PHM '10. pp. 1-8 (2010) 